\title{
Análise da condição corpórea, biometria externa e das vísceras do trato gastrointestinal de canários-da-terra, Sicalis flaveola braziliensis ${ }^{1}$
}

\author{
Raul A.S. Siqueira ${ }^{2 *}$, Arthur C.L. Lima², Tarsila Cavalcanti ${ }^{3}$, Paulo G.C. Wagner ${ }^{3}$ \\ e Ricardo R. Guerra ${ }^{2}$
}

\begin{abstract}
Siqueira R.A.S., Lima A.C.L., Cavalcanti T., Wagner P.G.C. \& Guerra R.R. 2013. [Analysis of body condition and external and gastrointestinal biometry of saffron finch, Sicalis flaveola braziliensis.] Análise da condição corpórea, biometria externa e das vísceras do trato gastrointestinal de canários-da-terra, Sicalis flaveola braziliensis. Pesquisa Veterinária Brasileira 33(3):379-383. Setor de Anatomia Animal, Universidade Federal da Paraíba, Centro de Ciências Agrárias, Areia, PB 58397-000, Brazil, E-mail: siqueiraras@gmail.com

Forty-one saffron finch, Sicalis flaveola brasiliensis, were studied regarding the external biometry, corporeal and plumage conditions, gastrointestinal tract (GIT) biometry, and the visceral topography, in order to provide morphological data and to characterize the condition in which these birds came to the wild animal screening Center. The visceral topography was similar to the found in parakeets and ostriches; however the last have a cecum. There was also relationship between the unfavorable body conditions and the loss of feathers. It was concluded that $S$. flaveola braziliensis has biometric measurements similar to other Passeriformes, however with differences to birds of the same gender, and few biometric differences among males and females. The results demonstrate that the corporal conditions of trafficked animals should be considered in wild animal screening in order to perform a better nutritional and clinical management, and to lower mortality.
\end{abstract}

INDEX TERMS: Sicalis flaveola brasiliensis, saffron finch, Passeriformes, birds, morphology, corporeal score, animal traffic.

RESUMO.- Analisaram-se em canários-da-terra, Sicalis flaveola brasiliensis, apreendidos pelo Cetas-IBAMA/PB e que morreram logo após sua chegada, as medidas biométricas externas, condições corpóreas e de plumagem, medidas biométricas das vísceras do trato gastrointestinal (TGI), assim como a topografia visceral, a fim de fornecer dados morfológicos e caracterizar as condições em esses pássaros chegaram a esse centro de triagem. A topografia visceral estava em consonância com a de periquitos e avestruz, a exceção que essa última espécie apresenta um ceco. Verificou-se que há relação entre as condições corpóreas desfa-

\footnotetext{
${ }^{1}$ Recebido em 12 de julho de 2012.

Aceito para publicação em 5 de novembro de 2012.

${ }^{2}$ Departamento de Medicina Veterinária, Centro de Ciências Agrárias (CCA), Universidade Federal da Paraíba (UFPB), Campus Universitário, Brejo Paraibano, Areia, PB 58397-000, Brasil. *Autor para correspondência: siqueiraras@gmail.com

${ }^{3}$ Centro de Triagem de Animais Silvestres-IBAMA/Paraíba (CETAS-IBAMA), Floresta Nacional da Restinga de Cabedelo, Estrada de Cabedelo, BR 230, Cabedelo, PB 58310-000, Brasil.
}

voráveis e a perda de plumagem. Conclui-se, que S. flaveola braziliensis possui medidas biométricas em consonância á de outros Passeriformes, contudo possui divergências para aves do mesmo gênero e poucas diferenças biométricas entre machos e fêmeas. Através do estudo, verifica-se que as condições corpóreas de animais traficados devem ser consideradas nos centros de triagem, a fim de se fazer um melhor manejo nutricional e/ou clínico, diminuindo a mortalidade.

TERMOS DE INDEXAÇÃO: Sicalis flaveola brasiliensis, canários-da-terra, Passeriformes, pássaros, morfologia, avaliação corpórea, tráfico de animais.

\section{INTRODUÇÃO}

O canário-da-terra (Sicalis flaveola) é um pássaro pertencente à ordem Passeriforme, subordem Oscine, caracterizados por aves com dimensões diminutas e siringe mais complexa e desenvolvida, concedendo-lhes maior capacidade e habilidade canora (Silveira \& Méndez 1999), despertando dessa forma, maior interesse pelos criadores e comer- 
ciantes. Levantamento realizado no Centro de Triagem de Animais Silvestres (Cetas)-IBAMA/PB realizado por Pagano (2009) demonstrou que $79 \%$ das aves apreendidas pertenciam à ordem de Passeriformes, número esse que exemplifica a predileção dos traficantes e, por conseguinte, dos consumidores por essas aves. Dentre elas, o canário-da-terra se destaca na lista como uma das espécies mais traficadas entre agosto de 2006 a julho de 2007, na qual 212 espécimes foram apreendidos no Cetas-IBAMA/PB (Renctas 2001).

Os canários-da-terra possuem entre $11-15 \mathrm{~cm}$ e apresentam marcado dimorfismo sexual (Ridgely \& Tudor 1989). São pássaros de formações abertas, forrageiam no solo ou próximo a ele (Sick 1997), e sua distribuição estende-se desde a América Central a América do Sul, com introdução no Havaí, Panamá, Porto Rico e Jamaica (Ridgely \& Tudor 1989). Sua alimentação é caracterizada por uma dieta granívora, incluindo algumas verduras, insetos e restos de ração de animais domésticos, quando há proximidade com estes (Machado 1980).

Existem quatro espécies e dez subespécies de canário-da-terra que se apresentam em populações dispersas e em abrangente distribuição: Sicalis citrina citrina, S. citrina browni, S. comlumbiana goeldii, S. comlumbiana leopoldinae, S. flaveola brasiliensis, S. flaveola pelzeni, S luteola luteola, S. luteola flavíssima, S. luteola chapmani, S. luteola luteiventris (Sick 1997, Rindgely \& Tudor 1989). Como não há trabalhos que caracterizem morfologicamente essas espécies e subespécies, existe a necessidade de estudos para a caracterização morfológica e taxonômica correta dos mesmos (Silveira \& Mendéz 1999).

Segundo Sick (1997), Sinbley \& Monroe (1990) e Silveira \& Mendéz (1999) as subespécies possuem as seguintes características: S. citrina citrina ou canário-da-horta que são aves menores, os quais o macho possui coloração amarelo-limão no alto da cabeça, asas marrom-escuro, dorso oliva com estrias e cauda marrom-escuro e a fêmea similar ao macho, pouco mais escura; S. citrina browni se assemelha a S. citrina citrina, porém menor; $S$. columbiana goeldii ou canário-do-amazonas que se assemelha a S. flaveola, contudo com dimensões reduzidas e ventre esbranquiçado, sem estrias; $S$. columbiana leopoldinae semelhante a $S$. columbiana goeldii, porém com tonalidades mais escuras e ventre acinzentado e estrias proeminentes na região peitoral; S. flaveola pelzeni ou canário-do-sul que apresenta plumagem similar ao de $S$. f. brasiliensis, contudo apresenta maior densidade de listras; $S$. luteola luteola na qual os machos possuem na região uropigiana uma coloração amarelo citrino e as fêmeas uma tonalidade amarelada nesta região, as bordas de suas penas dorsais são levemente amareladas e as fêmeas apresentam ainda o dorso bastante estriado chegando a formar um colar; S. luteola flavissima apresenta os lados da cabeça em machos amarelo oliva, ventre amarelado com peito amarelo oliva; S. luteola chapmani os machos possuem dorso amarelo esverdeado, ventre amarelo citrino brilhante sem tons de oliva na região peitoral; e S. luteola luteiventris ou canário-tipio que tem cabeça de cor amarelada com tonalidades menos marcadas e estria de cor cinza na região malar, peitoral acinzentada e ventre amarelo pálido.
Além das características externas, as medidas biométricas de uma espécie contribuem para traçar um padrão característico da espécie ou mesmo das subespécies, assim como relacionar e observar os aspectos evolutivos, fisiológicos, taxonômicos e ecológicos dos indivíduos (Bugoni et al. 2002). Esses estudos morfológicos ajudam na resolução da problemática do gênero Sicalis, ora locado na família Emberezidae (Howard \& Moore 1994), ora na família Fringillidae (Sinbley \& Monroe 1990), assim como na divergência quanto às subespécies.

As medidas que caracterizam um sistema biológico dizem respeito a tendência fisiológica deste, por exemplo, no aparelho digestório o comprimento é avaliado a fim de verificar a velocidade da passagem do alimento, sendo a maior extensão propícia para maior período de exposição do alimento às vilosidades intestinais (Cherry \& Siegel 1970). Entretanto, há escassez de dados biométricos referentes a ordem Passeriformes, não existindo tais mensurações da morfologia externa e dos órgãos do trato gastrointestinal (TGI) para as diferentes subespécies de canário-da-terra na literatura.

0 presente trabalho teve como objetivos verificar a qual subespécie pertencia os espécimes apreendidos, descrever morfologicamente o TGI dos canários-da-terra, comparando-o entre machos e fêmeas, estudar a topografia visceral da espécie, assim como, verificar as condições a que esses animais chegam no CETAS-PB. Tais resultados fornecerão subsídios para a classificação biométrica e taxonômica da espécie e para procedimentos clínicos e nutricionais em centros de triagem, criações comerciais ou ainda em programas de reprodução e reintrodução na natureza.

\section{MATERIAL E MÉTODOS}

Utilizaram-se quarenta e um espécimes, vinte e nove machos e doze fêmeas, de canários-da-terra oriundos de apreensões realizadas em 2010, por fiscais do Centro de Triagem de Animais Silvestres/IBAMA da cidade de Cabedelo/PB, e fornecidas ao Programa de Pós-Graduação em Ciência Animal, UFPB através do convênio número 02019.00129/2009-12. As aves vieram a óbito por motivos não relatados logo após chegarem ao CETAS, e foram acondicionadas sob refrigeração por um período máximo de uma semana até a análise das carcaças. Os espécimes foram pesados utilizando balança analítica (AY220, Marte ${ }^{\circledR}$, Brasil), mensurados com paquímetro digital (601-0150D, Caliper®, Taiwan).

As medidas mensuradas foram: comprimento total com penas, comprimento total sem penas, comprimento da cauda, comprimento dos tarsos, comprimento das asas com penas, comprimento das sem penas, comprimento das asas fechadas, comprimento da cabeça, largura da cabeça, altura do bico, largura do bico, comprimento do bico, comprimento do dedo médio com unha, comprimento do dedo médio sem unha, largura do tórax, largura do abdômen, comprimento do esôfago, comprimento do proventrículo, comprimento da moela, largura da moela, espessura da moela, comprimento do intestino e somatório de toda extensão do TGI, em consonância às medidas utilizadas por Bierregaard (1988) e da nomina padrão empregada às aves (Sick 1997, Baunmel, 1993).

As condições das aves foram classificadas e analisadas através da condição corpórea em Boa, Magra, Caquética e Obesa avaliando-se e a exposição esquelética, massa muscular e quantidade de tecido adiposo no dorso e demais extensões subcutâneas (San- 
chez 2008) e através da observação da perda ou não de plumagem. Na análise estatística compararam-se as médias oriundas da biometria entre machos e fêmeas aplicando-se o Teste de Mann-Whitney $(\alpha=0,05)$ (GraphPad Prisma 5).

\section{RESULTADOS}

Os espécimes estudados foram caracterizados como Sicalis flaveola braziliensis segundo as características externas encontradas. Fêmeas e machos tinham peso, respectivamente, de $12,82 \pm 0,16 \mathrm{~g}$ e 13,76 $\pm 0,27 \mathrm{~g}$. As medidas biométricas externas e do TGI das aves macho e fêmea se encontram respectivamente nos Quadros 1 e 2 .

Em relação á topografia visceral, o esôfago apresentou-se fino, sendo mais espesso na aproximação com a cavidade celomática, representando o esôfago glandular ou inglúvio, que está ventralmente posicionado à traqueia, adentrando na cavidade celomática, passando pela siringe (bem desenvolvida) e fundindo-se com o proventrículo. 0 proventrículo apresentou-se como uma porção fusiforme e mais escura, ventral aos pulmões, continuando com a moela. A moela está cranialmente posicionada ao lobo esquerdo hepático e caudalmente em comunicação com o intestino delgado. 0 fígado possui dois lobos, com coloração escura e está situado caudalmente ao coração. As alças intestinais se encontraram em contato com a moela. Houve ausência de vesícula biliar e ceco (Fig.1).
A análise das condições corpóreas demonstrou que $22 \%$ dos animais tinham condição corpórea Boa (peso: $13,76 \pm 1,31 \mathrm{~g}$ ) dos quais $33,3 \%$ apresentam perda de plumagem em pelo menos uma região do corpo, 61\% foram classificados como Magra (peso: 13,20 $2,80 \mathrm{~g}$ ) dos quais $68 \%$ apresentaram perda de penas. $7,3 \%$ foram classi-

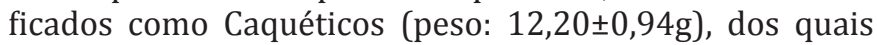
$100 \%$ apresentaram falta de plumagem. Os $9,8 \%$ restantes possuíam condição corpórea Obesa (peso: 14,23ะ5,17g) na qual $75 \%$ apresentaram falta de penas. Alguns animais com condições corpórea Caquética e Magra apresentaram lesões cutâneas (Quadro 3, Fig.2).

\section{DISCUSSÃO}

De acordo com as características externas descritas por Sick (1997), Sinbley \& Monroe (1990) e Silveira \& Mendéz (1999) os espécimes estudados foram classificados como Sicalis flaveola braziliensis. Na literatura são escassos os estudos biométricos com os Passeriformes silvestres. Em relação à biometria do TGI, nenhum membro do gênero Sicalis foi descrito. Entretanto, em relação à biometria externa, existe a descrição, embora parcial, de S. luteola (canário-da-horta), que vive nos estados do Norte do Brasil (Amapá, Pará e Roraima), Venezuela, Colômbia, Suriname e Guiana Francesa (Silveira \& Mendéz, 1999). Este é mais pesado que $S$. flaveola braziliensis do presente estudo (fêmea:

Quadro 1. Peso (g) e medidas biométricas externas (mm) de Sicalis flaveola braziliensis fêmeas e machos apreendidos pelo Cetas-IBAMA/PB em 2010. Média \pm Desvio Padrão, Máximo-Mínimo

\begin{tabular}{lcc}
\hline \multirow{2}{*}{ Medidas biométricas externas } & \multicolumn{2}{c}{ Fêmea } \\
\cline { 2 - 3 } & \multicolumn{2}{c}{$\mathrm{mm}$} \\
\hline Comp. total com penas & $117,07 \pm 4,62 ; 124,91-108,24$ & $119,02 \pm 11,11 ; 143,04-90,92$ \\
Comp. total sem penas & $67,79 \pm 5,27 ; 75,64-56,92$ & $70,70 \pm 6,90 ; 88,39-57,96$ \\
Comprimento da Cauda & $50,21 \pm 3,12 ; 55,83-45,09$ & $49,41 \pm 8,00 ; 62,22-28,99$ \\
Comprimento dos tarsos & $26,29 \pm 3,50 ; 30,60-20,56$ & $25,52 \pm 4,58 ; 34,31-17,26$ \\
Comp. da asa com penas & $101,86 \pm 5,60 ; 108,87-91,30$ & $103,13 \pm 11,72 ; 146,11-83,23$ \\
Comp. da asa sem penas & $50,31 \pm 5,75 ; 59,73-40,54$ & $49,61 \pm 5,42 ; 60,42-36,60$ \\
Comp. das asas fechadas & $68,27 \pm 2,42 ; 72,18-64,42 \mathrm{~b}$ & $70,29 \pm 10,48 ; 108,87-47,48$ a \\
Comprimento da cabeça & $17,30 \pm 1,13 ; 19,75-14,95 \mathrm{~b}$ & $18,74 \pm 2,41 ; 27,27-12,29$ a \\
Largura da cabeça & $13,39 \pm 0,76 ; 14,81-12,17$ & $13,66 \pm 1,24 ; 15,15-9,19$ \\
Altura bico & $7,85 \pm 0,90 ; 9,04-6,27$ & $7,66 \pm 0,97 ; 9,62-4,78$ \\
Largura do bico & $6,66 \pm 0,73 ; 8,39-5,63$ & $6,73 \pm 0,56 ; 7,90-5,79$ \\
Comprimento bico & $11,44 \pm 1,24 ; 13,37-9,34$ & $11,17 \pm 1,88 ; 14,28-7,21$ \\
Comp.do dedo médio com unha & $15,92 \pm 1,40 ; 18,75-13,97$ & $16,81 \pm 1,86 ; 19,97-11,53$ \\
Comp. do dedo médio sem unha & $11,95 \pm 0,95 ; 13,58-10,73 \mathrm{~b}$ & $13,09 \pm 1,75 ; 16,47-8,35$ a \\
Largura do tórax & $13,60 \pm 2,09 ; 18,72-11,16$ & $14,17 \pm 2,12 ; 21,57-10,17$ \\
Largura do abdômen & $13,38 \pm 1,04 ; 14,88-11,15$ & $14,69 \pm 1,75 ; 18,01-11,18$ \\
& & \\
Peso & $12,82 \pm 1,64 ; 15,07-9,69$ & $13,76 \pm 2,71 ; 21,49-8,99$
\end{tabular}

Quadro 2. Medidas biométricas do trato gastrointestinal (TGI) (mm) de Sicalis flaveola braziliensis fêmeas e machos apreendidos pelo Cetas-IBAMA/PB. Média \pm Desvio Padrão, Máximo-Mínimo

\begin{tabular}{lcc}
\hline \multicolumn{1}{c}{ Medidas biométricas do TGI } & Fêmea & Macho \\
\cline { 2 - 3 } & \multicolumn{2}{c}{$\mathrm{mm}$} \\
\hline Comprimento do esôfago & $36,00 \pm 6,81 ; 48,84-26,04$ & $39,65 \pm 5,37 ; 49,26-26,05$ \\
Comprimento do proventrículo & $12,67 \pm 2,10 ; 15,69-8,74$ & $11,98 \pm 2,94 ; 18,32-6,85$ \\
Comprimento moela & $14,17 \pm 1,95 ; 17,84-10,99$ & $12,88 \pm 2,13 ; 16,69-7,05$ \\
Largura da moela & $10,79 \pm 1,71 ; 14,62-8,46$ & $8,99 \pm 2,35 ; 12,45-4,56$ \\
Espessura da moela & $7,45 \pm 1,40 ; 10,05-5,46$ & $7,96 \pm 1,93 ; 12,23-5,00$ \\
Comprimento do Intestino & $203,06 \pm 28,94 ; 250,36-166,82$ & $191,50 \pm 25,44 ; 256,98-148,70$ \\
Somatório do comp. do TGI & $265,91 \pm 31,47 ; 311,29-224,75$ & $256,01 \pm 26,18 ; 324,96-202,03$
\end{tabular}



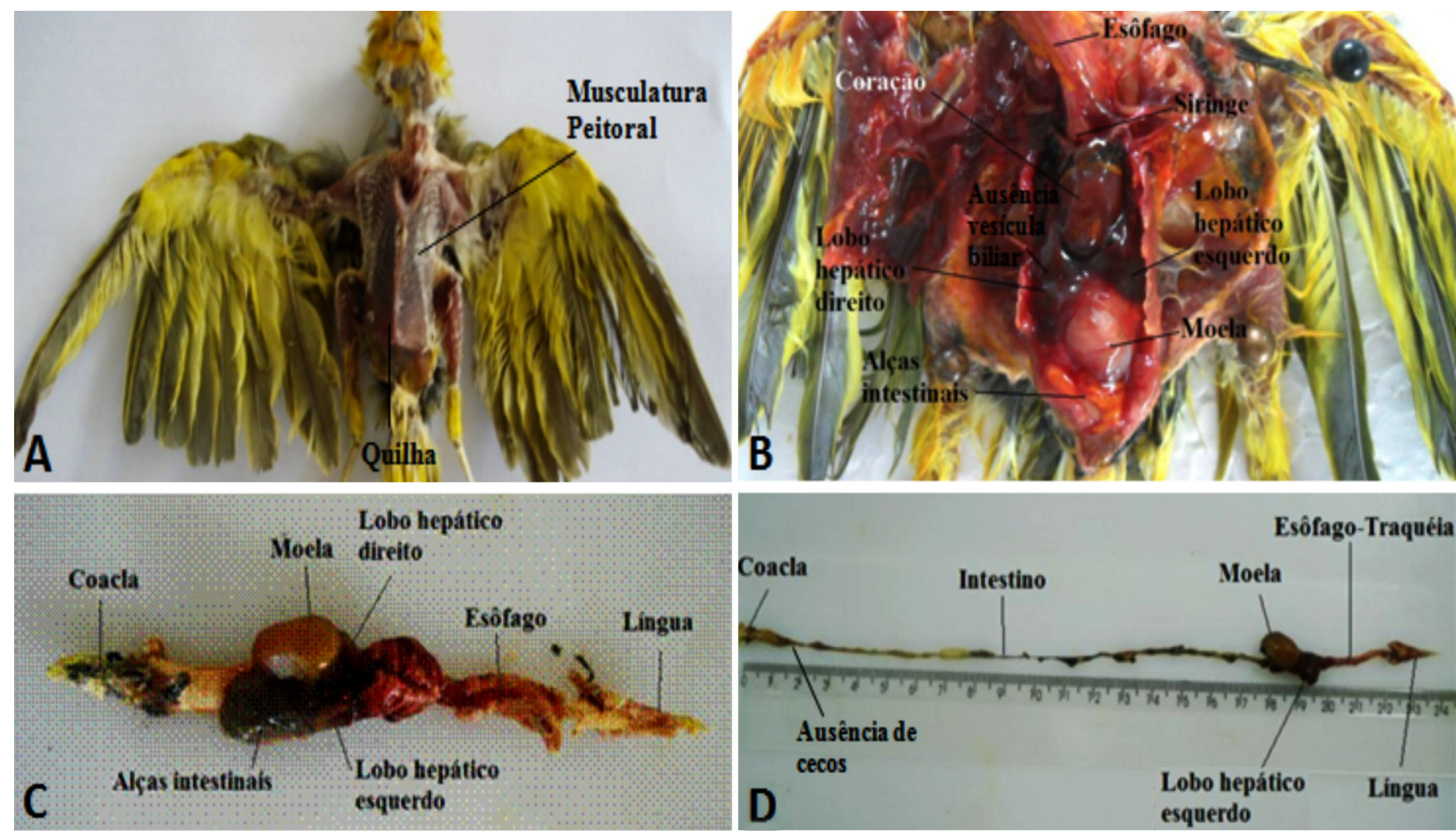

Fig.1. Sicalis flaveola braziliensis apreendidos pelo Cetas-IBAMA/PB. (A) Cavidade celomática fechada de um espécime classificado como magro. (B) Cavidade celomática aberta demonstrando a topografia dos órgãos. (C) Topografia das vísceras. (D) Trato gastrointestinal esticado.

Quadro 3. Peso (g) e desvio padrão (DP) de Sicalis flaveola braziliensis apreendidos pelo Cetas-IBAMA/PB para as diferentes classificações de condições corpóreas

\begin{tabular}{lcc}
\hline \multicolumn{1}{c}{ Condição corpórea } & Peso (g) & DP (\%) \\
\hline Boa & 13,76 & 1,31 \\
Magra & 13,2 & 2,8 \\
Caquética & 12,2 & 0,94 \\
Obesa & 14,23 & 5,17
\end{tabular}

$12,82 \pm 1,64 \mathrm{~g}$; macho: $13,76 \pm 2,71 \mathrm{~g}$ ) assim como outros Passeriformes, como o pula-pula-assoviador (Basileuterus leucoblepharus) $(16,90 \pm 1,00 \mathrm{~g})$ e a cigarrinha (Haplospiza unicolor) (16,30 $\pm 0,60 \mathrm{~g})$. Segundo Burgoni (2002), a fêmea de $S$. luteola $(16,00 \pm 2,00 \mathrm{~g})$ apresenta peso superior ao macho $(15,00 \pm 1,40 \mathrm{~g})$, contudo no presente estudo, o peso para S. flaveola braziliensis fêmea e macho não diferiram significativamente $(\alpha=0,05)$.

As medidas biométricas externas de fêmeas e machos dos espécimes de $S$. flaveola braziliensis diferiram significativamente apenas para comprimento de asas fechadas, comprimento de cabeça e comprimento de dedo médio sem unha. Dessa forma, nota-se que apesar do marcado dimorfismo sexual encontrado na coloração das penas dessa subespécie, biometricamente poucas diferenças são encontradas. As medidas biométricas do TGI não diferiram significativamente entre fêmeas e machos.

Comparando com outras espécies, a medida de comprimento de asa com penas de $S$. flaveola braziliensis são maiores que as encontradas em outros Sicalis como o S. luteola (fêmea $69,70 \pm 3,20 \mathrm{~mm}$; macho $74,00 \pm 3,50 \mathrm{~mm}$ ) e que ou-

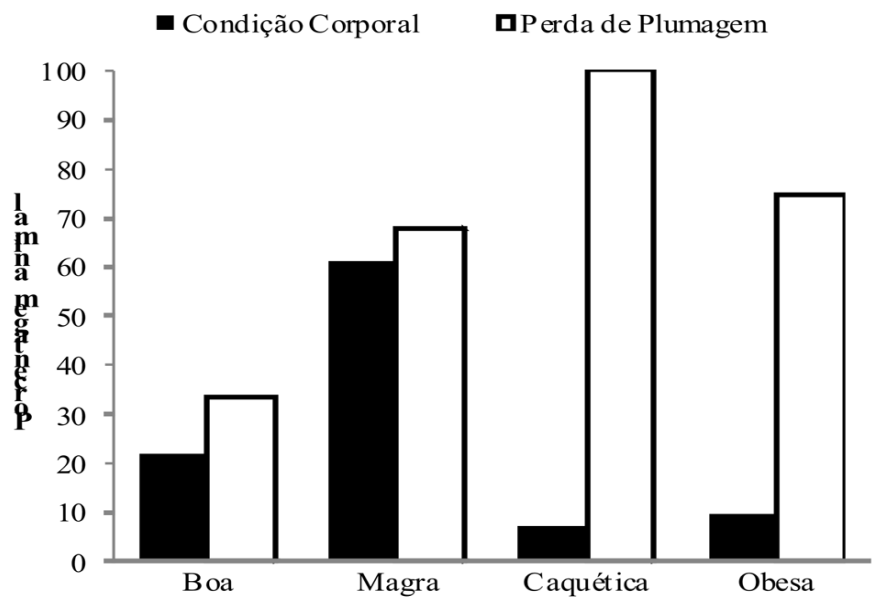

Fig.2. Gráfico demonstrando a condição corpórea e perda de plumagem (\%) de Sicalis flaveola braziliensis apreendidos pelo Cetas-IBAMA/PB.

tros Passeriformes como a cigarrinha $(60,00 \pm 2,60 \mathrm{~mm})$. Entretanto, as médias de $S$. flaveola braziliensis são semelhantes às encontradas para membros da família Muscicapidae: sabiá-coleira (Turdus albicollis) com 113,60 $\pm 4,10 \mathrm{~mm}$ e sabiá-poca (Turdus amaurochalinus) com 115,20 $\pm 3,70 \mathrm{~mm}$ (Burgoni 2002). 0 tarso ou tibiotarso é um osso que pode variar bastante, inclusive entre as linhagens ou subespécies como é observado em galinhas (Gallus gallus domesticus) (Getty 1981). S. flaveola braziliensis apresentou comprimento dos tarsos maior que $S$. luteola (fêmea $15,00 \pm 2,60 \mathrm{~mm}$, macho $16,40 \pm 2,10 \mathrm{~mm}$ ) apesar de este apresentar maiores dimensões corpóreas. S. flaveola bra- 
ziliensis apresentou comprimento dos tarsos semelhante ao da noivinha (Xolmis irupero) $(25,00 \mathrm{~mm})$ e com o choca de chapéu-vermelho (Thomnophilus ruficapillus) (fêmea 26,00 $\pm 0 \mathrm{~mm}$ e macho 27,20 $\pm 0,60 \mathrm{~mm}$ ) (Burgoni 2002).

Medidas biométricas do TGI descritas na literatura para aves são escassas. Já as dimensões para proventrículo são quase ausentes na literatura, sendo o comprimento em galinhas de 50mm (Mcleod 1964). Em S. flaveola braziliensis os valores para fêmeas e machos foram respectivamente de $12,67 \pm 2,10 \mathrm{~mm}$ e $11,98 \pm 2,94 \mathrm{~mm}$. Segundo Turk (1982), o comprimento do trato entérico é maior em aves herbívoras e menores nas carnívoras, com base nessa afirmativa observou que o comprimento do trato intestinal de $S$. flaveola braziliensis foi relativamente grande (fêmea: $265,91 \pm 31,47$ e macho: $256,01 \pm 26,18$ ) se comparado ao de outras espécies domésticas estudadas, como o pato doméstico (Anas platyrhynchos) $155-233 \mathrm{~cm}$ e o ganso (Anser anser) 250-365 cm (Pilz 1937), levando em conta as proporções de peso e tamanho das espécies. 0 tamanho do TGI e a relação TGI/tamanho corpóreo $(1,81)$ de $S$. flaveola está em consonância com a obtida para galos-de-campina $(1,84)$ (França et al. 2010).

As medidas biométricas descritas no presente estudo são as primeiras na literatura para S. flaveola braziliensis e poderão viabilizar a correta taxonomia do $S$. flaveola contribuindo para a sistemática dos Passeriformes.

Quando analisada a topografia visceral do TGI de $S$. flaveola braziliensis, observou-se que a mesma está em consonância com a de outras aves domésticas (Getty 1981) e de periquitos-australianos (Matsumoto 2009). A ausência de cecos e vesícula biliar observada é comum entre as espécies do gênero (Sick 1997).

No que se refere às condições corpóreas, Wolf (2003), descreveu existência de variação relevante no peso de espécies Passeriformes (14\%), que é superior a de Columbiformes $(7,4 \%)$, confirmando a suscetibilidade dos Passeriformes para déficit nutricional. Levantamentos sobre as causas de mortalidade em pássaros de vida livre traficados demonstram que $47,62 \%$ das mortes advêm de distúrbios nutricionais, e que $40,70 \%$ dos Passeriformes traficados que morem tem condição corpórea magra e $19,77 \%$ condição caquética (Sanches 2008). Esses resultados corroboram com os dados apresentados no presente estudo, no qual se observa uma alta incidência de animais com condição corpórea magra e caquética, o que pode estar relacionado com problemas nutricionais e com a morte dos mesmos. Mesmo os animais considerados obesos podem ser resultados de negligência nutricional ou falta de exercício físico por falta de espaço em condição de cativeiro, além de possuírem relação direta com a perda de penas (75\%).

\section{CONCLUSÕES}

Conclui-se que Sicalis flaveola braziliensis possui algumas divergências biométricas para as aves do mesmo gênero e poucas diferenças biométricas entre machos e fêmeas, sendo essas descrições importantes para viabilizar a correta taxonomia desta espécie contribuindo para a sistemática dos Passeriformes.

Os resultados também demonstram que as condições corpóreas de animais traficados devem ser consideradas nos centros de triagem a fim de se realizar um melhor manejo nutricional e/ou clínico diminuindo, dessa forma, a mortalidade.

Agradecimentos.- Ao Cetas-IBAMA/PB pelo fornecimento das aves e ao CNPq pela concessão de bolsa de Iniciação Científica.

\section{REFERÊNCIAS}

Baumel J.J., King A.S., Breazile J.E., Evans H.E. \& Vanden B.J.C. 1993. Handbook of avian anatomy: Nomina anatomica avium. $2^{\text {nd }}$ ed. Nuttall Ornithological Club, Cambridge, Massachussets.

Bierregaard Jr R.O. 1988. Morphological data from understory in terra firme forest in the Central Amazonian Basin. Revta. Brasil. Biol. 48:169-178.

Burgoni L., Mohr L.V., Scherer A., Efe M.A. \& Scherer S.B. 2002. Biometry, moult and brood patch parameters of birds in Southern Brazil. Revta Bras. Ornitol. 10(1):85-94.

Cherry J.A. \& Siegel P.B. 1978. Selection for body weight of age: Feed passage and intestinal size of normal and dwarf chicken. J. Poult. Sci. 57(2):336-340.

Dukes G.E. 1996. Fisiologia dos Animais Domésticos. Guanabara-Koogan, Rio de Janeiro.

França E.C.S., Lima A.L.T.M., Siqueira R.A.S., Luna A.C., Oliveira Neto T.S., Barreiro D.C. \& Guerra R.R. 2010. Biometria externa e do trato gastrointestinal do galo-de-campina (Paroaria dominicana, Linnaeus). Anais II Semevet, Areia. (Resumo)

Getty R. 1981. Anatomia dos Animais Domésticos. 5ํㅡ ed. Interamericana, Rio de Janeiro.

Howard R. \& Moore A. 1994. A complete checklist of the birds of the world. $2^{\text {nd }}$ ed. Academic Press, London. 630p.

Machado L.O.M. 1980. Alguns aspectos do comportamento e da biologia de Sicalis flaveola (Linnaeus, 1766) (Passeriformes: Emberezidae). USP, São Paulo.

Matsumoto F.S., Carvalho A.F., Franciolli A.L.R., Favaron P.O., Miglino M.A. \& Ambrósio C.E. 2009. Topografia e morfologia das vísceras do periquito-australiano (Melopsittacus undulatus Shaw, 1805). Ciênc. Anim. Bras. 10(4):1263-1270.

Mcleod W.M., Trotter D.M. \& Lumb J.W. 1964. Avian Anatomy. Burgess Publ., Minneapolis.

Pagano I.S.A., Sousa A.E.B.A., Wagner P.G.C. \& Ramos R.T.C. 2009. Aves depositadas no Centro de Triagem de Animais Silvestres do IBAMA na Paraíba: uma amostra do tráfico de aves silvestres no estado. Ornithologia 3(2):132-144.

Pilz H. 1937. Merkmale am Darmkanal des Hausgeflügels (Gans, Ente, Huhn, Taube). Gegenbaurs Morphol. Jahrb. 79(1):275-304.

Renctas 2001. 1ำ Relatório Nacional sobre o Tráfico de Fauna Silvestre. Rede Nacional de Combate ao Tráfico de Animais Silvestres, Brasília. 108p. (Apud Pagano et al. 2009)

Ridgely R.S. \& Tudor G. 1989. The Birds of South America: The Oscine passerines. Vol.1. University of Texas Press, Austin. 516p.

Sanches T.C. 2008. Causas de morte em passeriformes: comparação entre aves de vida livre residentes da região metropolitana de São Paulo e aves oriundas do tráfico. FMVZ-USP, São Paulo.

Sibley C.G. \& Monroe Jr B.L. 1990. Distribution and Taxonomy of the Birds of the World. Yale Univ. Press, New Haven,. 1111p.

Sick H. 1997. Ornitologia Brasileira. Nova Fronteira Rio de Janeiro. 862p.

Silveira L.F. \& Mendéz A.C. 1999. Caracterização das formas brasileiras do gênero Sicalis (Passeriformes: Emberezidae). Atualidades Ornitológicas, Ivaiporã/PR, 90:6-8.

Turk D.E. 1982. The anatomy of the avian digestive tract as related to feed utilization. J. Poult. Sci. 61(7):1225-1244.

Wolf P., Rabehl N. \& Kamphues J. 2003. Investigations on feathering, feather growth and potential influences of nutrient supply on feathers regrowth in small pet birds (canaries, budgerigars and lovebirds). J. Anim. Physiol. Anim. Nutr. 87:134-141. 